\title{
Role of Recombinant Human Granulocyte-Macrophage Colony-Stimulating Factor (rhGM-CSF) in Wound Healing in Critical Care Settings
}

\author{
Abdulmajeed Moharag ${ }^{1}$, Albatool Mansouri², Bashayer Althaqafi², Julnar Halawani², Haneen Banjar ${ }^{3}$, Muna Alnaim $^{4}$ \\ ${ }^{1}$ Plastic Surgery and Burn Unit, King Fahad General Hospital, Jeddah, Saudi Arabia \\ ${ }^{2}$ College of Medicine, Umm Al-Qura University, Mecca, Saudi Arabia \\ ${ }^{3}$ Department of General Surgery, King Abdullah Medical City, Mecca, Saudi Arabia \\ ${ }^{4}$ College of Medicine, King Faisal University, Al Ahsaa, Saudi Arabia
}

Correspondence should be addressed to Abdulmajeed Moharag, Plastic Surgery and Burn Unit, King Fahad General Hospital, Jeddah, Saudi Arabia

Email: abalmajd1@gmail.com

Received: 28 August 2021, Revised: 28 August 2021, Accepted: 29 August 2021, Published: 31 August 2021

Copyright (C) 2021 Moharag et al. This is an open access article distributed under the Creative Commons Attribution License, which permits unrestricted use, distribution, and reproduction in any medium, provided the original work is properly cited.

\begin{abstract}
Surgical management for burns has been reported to effectively manage such cases via early excisions and grafting to enhance re-epithelization. However, the operation is very expensive and not be suitable for many patients, especially those suffering from extensive body burns across huge surface areas. Recombinant human granulocyte-macrophage colonystimulating factor has been validated to effectively manage severe burns, resulting in favorable outcomes and minimal or absent adverse events. Recombinant human granulocyte-macrophage colony-stimulating factor should be used for the management of such patients due to the favorable events that have been extensively reported among studies in the literature. Some of these events include reducing the healing time and increasing the rates of wound healing among burns patients, when compared to other treatment modalities. Additionally, various studies have also demonstrated that no significant adverse events are associated with it, adding to its efficacy in the management of deep burns. However, it should be noted that further research is still encouraged to further validate the current evidence, and to validate the effect on scarring. Further details are discussed within the manuscript.
\end{abstract}

Keywords: - Granulocyte stimulating factor; rhGM-CSF; burns; management; efficacy. 


\section{Introduction}

Burns are serious events that can have a significant threat to the affected patient's life, particularly for patients living in low socio-economic countries (1). The clinical significance of burns and risk of increased morbidity and mortality is more significant for severe burns, which usually require critical care. The increased mortality risk among these patients is usually attributable to the abundant release of cytokines and inflammatory mediators which can impair the different physiological processes within the human body (2). A reduced quality of life of the affected patients is also a potential outcome, especially in cases of severe and deep burns which usually damage the epidermal and dermal reticular layers leading to hypertrophic scar formation and skin deformities $(3,4)$. Reduced natural immunological functions of the skin may also facilitate bacterial invasion leading to more complications such as ulcerations and even sepsis (5). Therefore, approaches to the care of such events have been intended to effectively manage these patients to enhance the outcomes and improve the quality of life.

Surgical management has been reported to effectively manage these cases via early excisions and grafting to enhance re-epithelization (6-8). However, the operation is very expensive and not suitable for many patients, especially those suffering from extensive body burns across huge surface areas (9). Among the medical management modalities that have been proposed, recombinant human granulocyte-macrophage colonystimulating factor (rhGM-CSF) has been validated to effectively manage severe burns with favorable outcomes and minimal to absent adverse events (9). The favorable effects are usually attributed to its efficacy in promoting the proliferation of the hematopoietic progenitor cell within the bone marrow in addition to its role in mature cells transfer to the peripheral blood. It can also enhance the immune response to burns and promotes wound healing as a result of inducing the proliferation of monocytes and macrophages within the affected tissues (Figure 1) (10-13). Many studies in the current literature have evaluated the efficacy and safety of rhGM-CSF in promoting wound healing in critical care settings. In the present literature review, we aim to discuss the role of rhGM-CSF in wound healing in the critical care setting.

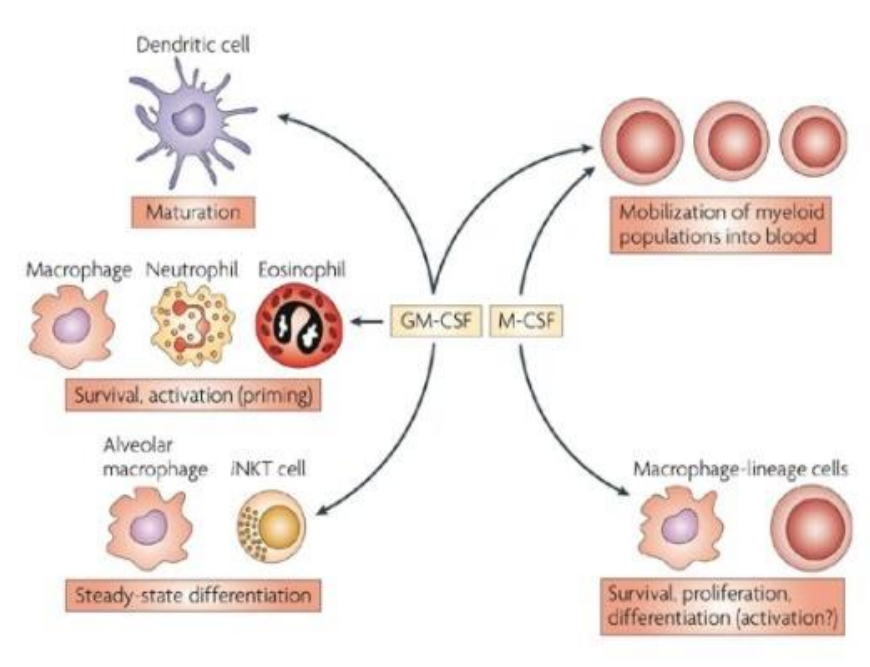

Figure 1. Main immunological functions of GM-CSF (14).

\section{Physiology and healing effect}

Researchers from different clinical settings have recently focused on the effects of rhGM-CSF in managing burns and wound healing (15). Many studies in the literature have been identified as reporting the effects of applying rhGM-CSF for the management of severe burns and wound healing. The physiological process of wound healing is a complicated one, as even after achieving the intended management outcomes for the affected patients, these wounds may be further complicated by unexpected adverse events such as inflammation and infections (16, 17). In critical care settings, rhGM-CSF has been effectively introduced as an efficacious modality for the management of extensive deep wounds (9).

Studies have indicated that the application of the modality has been associated with an outstanding performance for wound healing because it has a dual effect on the process of wound healing, significantly for infected wounds, via the induction of the process of healing and stimulation and modulation of the underlying patient's immunity leading to further protection against any potential infection (18). Improved immune potency was reported as the rhGMCSF action, which enables the modality to overcome bacterial infections and improve wound healing (13). 


\section{Journal of Healthcare Sciences}

For example, a previous investigation by $\mathrm{Yu}$ et al. (19) indicated that the administration of rhGM-CSF was effectively associated with the restoration of interleukin2 (IL-2) and T-cell functions, which was in turn associated with a reduction in the sepsis status, which is a generalized inflammatory condition of the whole body cells. Another investigation by Yuan et al. (9) compared the efficacy of rhGM-CSF versus mupirocin ointment. The authors of the study reported that the healing time within the mupirocin ointment group was significantly longer than the healing time that has been observed with the rhGM-CSF group $(23.8 \pm 4.6$ versus $17.28 \pm 6.70$ days), indicating the superior efficacy of rhGM-CSF administration for wound healing. In this context, another clinical trial by Yan et al. in 2017 also compared the effectiveness of rhGM-CSF administration on wound healing against a placebo group. The authors indicated that the effectiveness of rhGM-CSF was significantly better than the effects obtained within the placebo group as they found that the healing rates and time were both better within the treatment group.

The estimated time of wound healing was $18.8 \pm 7.6$ days for the rhGM-CSF group, and $25.5 \pm 4.6$ days for the placebo group (20). Furthermore, the authors reported that favorable effects of rhGM-CSF on wound healing is obtained via the effects on the migration and promotion of the keratinocytes and epithelial cells that can be variously found within the inflammation area. In addition, favorable effects were also found by the induction of the differentiation and proliferation of the hematopoietic progenitor cells into eosinophils, neutrophils, and macrophages. Following this, it has been observed that the stimulated keratinocytes and epithelial cells significantly promote the production of a neuroepithelial layer as a result of the process of re-epithelization (18).

A further investigation by Fang et al. (11) also demonstrated the efficacy of the exogenous administration of rhGM-CSF on wound healing in diabetic mice. They reported that the modality significantly induces the tissue infiltration of the neutrophils and macrophages as well as increase the levels of cytokines, which play a significant role in neovascularization (21).
Differentiation of the vascular endothelial cells and promotion and induction of neovascularization were also reported in an investigation by Mann et al. (12) as a result of the administration of rhGM-CSF.

The same observations were also indicated by Yan et al. (20), who indicated the favorable events that occur following rhGM-CSF administration. The authors of this latter study found that rhGM-CSF could enhance vascularity and increase the rate of blood capillaries formation more significantly than the placebo group.

Angiogenesis and re-epithelization were also reported to be significantly improved following the administration of rhGM-CSF, which further indicates that rhGM-CSF significantly enhances the process of rapid wound healing (20). Similarly, Liu et al. conducted a clinical trial to compare the safety and efficacy of rhGM-CSF against control groups that were treated for deep burns using conventional management modalities (22).

They reported that the time of wound healing was significantly longer in the control group than in the rhGMCSF group. Additionally, they showed that $96.6 \%$ and $88.95 \%$ in the rhGM-CSF and conventional therapy groups were completely cured following the administration of the interventions for each group, respectively.

Finally, the authors indicated that the rates of adverse drug events did not significantly differ between the two treatment groups, and therefore, the authors concluded that rhGM-CSF administration is an effective valid modality that should be used for the management of deep wounds.

In another clinical trial Liu et al. (23) also concluded that the rate and duration for achieving favorable debridement of the burnt wound significantly favored the administration of rhGM-CSF over conventional management modalities. The same findings were also reported in another trial by Wang et al. (24), which showed that the rate of wound healing, duration of healing, and the total effective rates all significantly favored the administration of rhGM-CSF. 


\section{Efficacy and outcome}

To assess the efficacy of rhGM-CSF, safety must also be evaluated as it can significantly impact patients' outcomes. Among the different studies in the literature, some have evaluated the safety of the compound when applied to manage extensive burns. Most of the included studies did not report any significant adverse events or complications following the administration of rhGM-CSF (9, 13, 20, 22-25). However, some adverse events and application-site reactions were observed among some patients in some studies. For instance, the investigation by Liu et al. (23) reported that five of their patients that were treated for their wounds with rhGM-CSF had more swollen and redder skin than observed in the patients in the control group. Moreover, they also indicated that three patients had simultaneous painful sensations around the wound area, and another two patients had pain, swelling, and slight redness. A further clinical trial by Liu et al. (22) also reported that a total of 44 patients out of their 2257 included population developed some adverse events, which primarily included painful sensations at the wound site. It is worth mentioning that other studies validated the administration of rhGM-CSF for other uses than wound management, like idiopathic pulmonary alveolar proteinosis, metastatic melanoma, and for critically-ill patients with impaired neutrophil phagocytosis, and no significant adverse events were reported among these studies (26-28). This indicates that rhGM-CSF is safe, in addition to being significantly effective, and therefore, its use should be encouraged in the future and be more implied in a wider clinical field.

A meta-analysis by Breederveld et al. (29) concluded that using recombinant human growth factors was significantly associated with reduced healing duration when compared to the control group. Furthermore, although no adverse events or infections were noticed, they reported that hyperglycemic was significantly higher in the growth factor adult population than the control group, while this significance was absent among the pediatric population. This was further indicated in the updated review by the same authors in 2014 (30). In a clinical trial by Wang et al. (24), the authors reported that the duration of healing was significantly longer in the control group than in the rhGM-CSF one, and the estimated median rate of healing significantly increased at $8,14,20$, and 28 days from the inauguration of the studies (Figure 2).

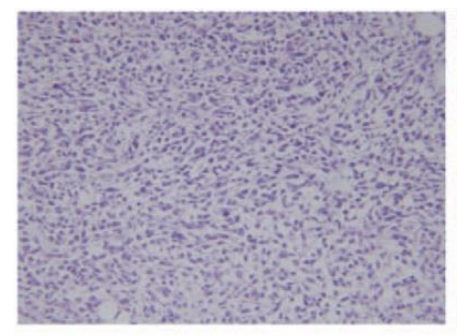

O DAY

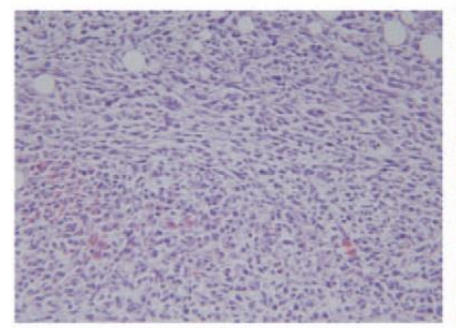

O DAY

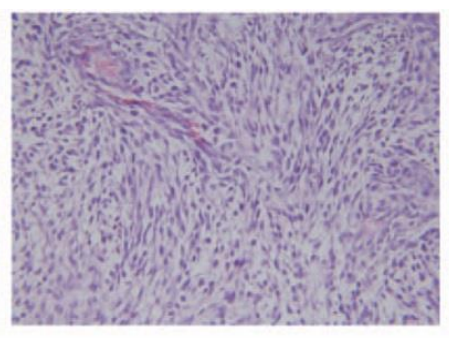

$14^{\text {th }}$ DAY

Placebo

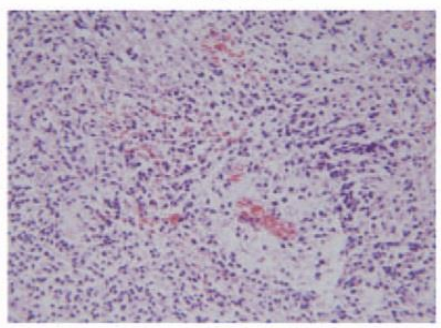

$14^{\text {th }}$ DAY

rhGM-CSF

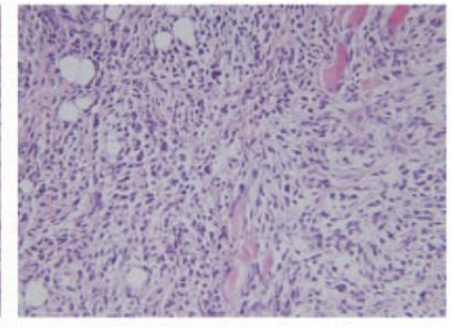

$28^{\text {th }}$ DAY

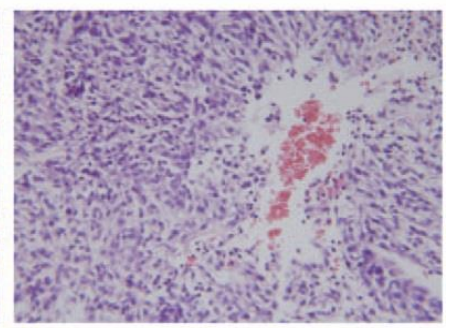

$28^{\text {th }}$ DAY

Figure 2. Wound tissues on hematoxylin-eosin stain treated with rhGM-CSF and placebo showing more significant angiogenesis in the granulation tissues at the different time points (20). 


\section{Journal of Healthcare Sciences}

In 2020, a meta-analysis was published by $\mathrm{Li}$ et al. (31) that analyzed the results of seven randomized controlled trials with 982 included patients who had suffered from excessive burns. They indicated that the use of rhGMCSF was significantly associated with a reduced healing time for burns in the treatment group patients, when compared to the control groups. Additionally, they also estimated that the rate of wound healing within the rhGMCSF group was significantly higher than in the control group from the time the trials were inaugurated and at 7 , 10,14 , and 20 days.

On the other hand, the pooled analysis by the authors showed that no significant difference within the healing time was noticed between the control and the rhGM-CSF groups at 28 days from the initiation of the included trials. A further identified outcome was the non-significant difference between the rhGM-CSF and the control groups in terms of mean vascular endothelial growth factor (VEGF) and fibroblast growth factor (FGF) from the day when the trials began, up to day 14 (31). This may be attributable to many factors, including that rhGM-CSF efficacy is only limited to shortening the healing period and that the modality is most effective within the earliest phases of the disease while the efficacy may decrease during the late stages.

However, no evidence regarding this information has been validated in the literature. Accordingly, further research must be encouraged to further investigate which factor is responsible for wound healing regulation across the different time intervals following the administration of rhGM-CSF for managing severe burns, as it is difficult to draw solid evidence and conclusions based on the findings from the present investigations in the literature. Scarring is not also adequately reported and investigated among studies, and therefore, future investigations should consider this point for further validation of the effects of rhGM-CSF when managing severe burns.

However, it should be noted that in the previous investigation by Yan et al. (20), they used the Vancouver scar scale of pigmentation, and the results indicated that rhGM-CSF had significantly superior scores than that of the control group. Despite this, further evidence could not be obtained from other studies, and therefore, it is difficult to make a solid conclusion.

\section{Conclusion}

Recombinant human granulocyte-macrophage colonystimulating factor should be used for the management of moderately to severe burn patients due to the favorable events that have been extensively reported among studies in the literature. Some of these events include reducing the healing time and increasing the rates of wound healing among burns patients as compared to other treatment options. Additionally, different studies have also demonstrated that no significant adverse events are associated with the administration of this method, adding to its efficacy in the management of deep burns. However, it should be noted that further research is still required to further validate the current evidence, validate the treatments's effect on scarring, and to further understand the effects on vascular endothelial growth factor and fibroblast growth factor.

\section{Disclosure}

\section{Statement:}

The authors declare no conflict of interest.

\section{Funding:}

None.

\section{Ethical Consideration:}

Non applicable.

\section{Data Availability:}

All data are presented within the paper.

\section{References}

1. Smolle C, Cambiaso-Daniel J, Forbes AA, Wurzer P, Hundeshagen G, Branski LK, et al. Recent trends in burn epidemiology worldwide: A systematic review. Burns : journal of the International Society for Burn Injuries. 2017;43(2):249-57. 


\section{Journal of Healthcare Sciences}

2. Liu LY, Hou YS, Chai JK, Hu Q, Duan HJ, Yu YH, et al. Basic fibroblast growth factor/vascular endothelial growth factor in the serum from severe burn patients stimulates the proliferation of cultured human umbilical cord mesenchymal stem cells via activation of Notch signaling pathways. The journal of trauma and acute care surgery. 2013;75(5):789-97.

3. Hu Z, Chen B, Li Y, Jiang W, Wen L, Ji F, et al. [Effect of tranilast on wound healing and administration time on scar hyperplasia of deep partial-thickness burn in mice]. Zhongguo xiu fu chong jian wai ke za zhi = Zhongguo xiufu chongjian waike zazhi $=$ Chinese journal of reparative and reconstructive surgery. 2017;31(4):465-72.

4. Oh H, Boo S. Quality of life and mediating role of patient scar assessment in burn patients. Burns : journal of the International Society for Burn Injuries. 2017;43(6):1212-7.

5. Li ZQ, Wang JH, Wu Q, Yang L. [Treatment of a patient with massive unhealed wound accompanied by wound sepsis in late stage after burn injury]. Zhonghua shao shang za zhi $=$ Zhonghua shaoshang zazhi $=$ Chinese journal of burns. 2012;28(6):458-61.

6. Engrav LH, Heimbach DM, Reus JL, Harnar TJ, Marvin JA. Early excision and grafting vs. nonoperative treatment of burns of indeterminant depth: a randomized prospective study. J Trauma. 1983;23(11):1001-4.

7. Muangman P, Deubner H, Honari S, Heimbach DM, Engrav LH, Klein MB, et al. Correlation of clinical outcome of integra application with microbiologic and pathological biopsies. J Trauma. 2006;61(5):1212-7.

8. Akita S, Akino K, Imaizumi T, Hirano A. Basic fibroblast growth factor accelerates and improves seconddegree burn wound healing. Wound repair and regeneration : official publication of the Wound Healing Society [and] the European Tissue Repair Society. 2008;16(5):635-41.

9. Yuan L, Minghua C, Feifei D, Runxiu W, Ziqiang L, Chengyue M, et al. Study of the use of recombinant human granulocyte-macrophage colony-stimulating factor hydrogel externally to treat residual wounds of extensive deep partial-thickness burn. Burns : journal of the International Society for Burn Injuries. 2015;41(5):1086-91.
10. Kaplan G, Walsh G, Guido LS, Meyn P, Burkhardt RA, Abalos RM, et al. Novel responses of human skin to intradermal recombinant granulocyte/macrophagecolony-stimulating factor: Langerhans cell recruitment, keratinocyte growth, and enhanced wound healing. The Journal of experimental medicine. 1992;175(6):1717-28.

11. Fang Y, Shen J, Yao M, Beagley KW, Hambly BD, Bao S. Granulocyte-macrophage colony-stimulating factor enhances wound healing in diabetes via upregulation of proinflammatory cytokines. The British journal of dermatology. 2010;162(3):478-86.

12. Mann A, Breuhahn K, Schirmacher P, Blessing M. Keratinocyte-derived granulocyte-macrophage colony stimulating factor accelerates wound healing: Stimulation of keratinocyte proliferation, granulation tissue formation, and vascularization. The Journal of investigative dermatology. 2001;117(6):1382-90.

13. Zhang L, Chen J, Han C. A multicenter clinical trial of recombinant human GM-CSF hydrogel for the treatment of deep second-degree burns. Wound repair and regeneration : official publication of the Wound Healing Society [and] the European Tissue Repair Society. 2009;17(5):685-9.

14.Hamilton JA. Colony-stimulating factors in inflammation and autoimmunity. Nature Reviews Immunology. 2008;8(7):533-44.

15. Chi YF, Chai JK, Luo HM, Zhang QX, Feng R. Safety of recombinant human granulocyte-macrophage colonystimulating factor in healing pediatric severe burns. Genetics and molecular research : GMR. 2015;14(1):2735-41.

16. Fujii T. Local treatment for extensive deep dermal thickness burn and follow-up study. Acta chirurgiae plasticae. 1990;32(1):46-56.

17. Barret JP, Herndon DN. Effects of burn wound excision on bacterial colonization and invasion. Plast Reconstr Surg. 2003;111(2):744-50; discussion 51-2.

18. Hu X, Sun H, Han C, Wang X, Yu W. Topically applied rhGM-CSF for the wound healing: a systematic review. Burns : journal of the International Society for Burn Injuries. 2011;37(5):729-41. 


\section{Journal of Healthcare Sciences}

19. Yu C, Wang J, Fu Y, Mao Y, Chen Y, Jiang Y, et al. Treatment of skin injury due to vinorelbine extravasation using bFGF and rhGM-CSF: an experimental study in a murine model. Biological research for nursing. 2011;13(1):32-7.

20. Yan D, Liu S, Zhao X, Bian H, Yao X, Xing J, et al. Recombinant human granulocyte macrophage colony stimulating factor in deep second-degree burn wound healing. Medicine. 2017;96(22):e6881.

21. Baker EA, Leaper DJ. Proteinases, their inhibitors, and cytokine profiles in acute wound fluid. Wound repair and regeneration : official publication of the Wound Healing Society [and] the European Tissue Repair Society. 2000;8(5):392-8.

22. Liu J, Liao ZJ, Zhang Q. [Phase IV clinical trial for external use of recombinant human granulocytemacrophage colony-stimulating factor gel in treating deep partial-thickness burn wounds]. Zhonghua shao shang za zhi $=$ Zhonghua shaoshang zazhi $=$ Chinese journal of burns. 2016;32(9):542-8.

23. Liu L, Fang Y, Yao M, Yu W, Li X. [Effect of recombinant human granulocyte-macrophage colonystimulating factor on wound debridement and healing of deep II thickness burn]. Zhongguo xiu fu chong jian wai ke za zhi $=$ Zhongguo xiufu chongjian waike zazhi = Chinese journal of reparative and reconstructive surgery. 2011;25(9):1059-62.

24. Wang ZY, Zhang Q, Liao ZJ, Han CM, Lv GZ, Luo $\mathrm{CQ}$, et al. [Effect of recombinant human granulocytemacrophage colony stimulating factor on wound healing in patients with deep partial thickness burn]. Zhonghua shao shang za zhi $=$ Zhonghua shaoshang zazhi $=$ Chinese journal of burns. 2008;24(2):107-10.
25. Yan $\mathrm{H}$, Chen J, Peng X. Recombinant human granulocyte-macrophage colony-stimulating factor hydrogel promotes healing of deep partial thickness burn wounds. Burns : journal of the International Society for Burn Injuries. 2012;38(6):877-81.

26. Ding J, Xiao Y, Dai J, Miao L, Qiu Y, Chen L, et al. [Effectiveness and safety of inhaled granulocytemacrophage colony-stimulating factor therapy in idiopathic pulmonary alveolar proteinosis]. Zhonghua yi xue za zhi. 2015;95(34):2766-70.

27. Kwek SS, Kahn J, Greaney SK, Lewis J, Cha E, Zhang L, et al. GM-CSF and ipilimumab therapy in metastatic melanoma: Clinical outcomes and immunologic responses. Oncoimmunology. 2016;5(4):e1101204.

28. Pinder EM, Rostron AJ, Hellyer TP, RuchaudSparagano MH, Scott J, Macfarlane JG, et al. Randomised controlled trial of GM-CSF in critically ill patients with impaired neutrophil phagocytosis. Thorax. 2018;73(10):918-25.

29. Breederveld RS, Tuinebreijer WE. Recombinant human growth hormone for treating burns and donor sites. The Cochrane database of systematic reviews. 2012;12:Cd008990.

30. Breederveld RS, Tuinebreijer WE. Recombinant human growth hormone for treating burns and donor sites. The Cochrane database of systematic reviews. 2014;2014(9):Cd008990.

31. Li J, Liu W, Zhang G, Wang D, Lou H, Duang J. Effectiveness of recombinant human granulocyte macrophage colony-stimulating factor for treating deep second-degree burns: a systematic review and metaanalysis. BMJ military health. 2020;166(5):352-7. 